\title{
Youngest reported case of liver injury related to Ashwagandha
}

\author{
Simran Waller ${ }^{1}$, Tsz Ho Wong ${ }^{1}$, and Robert Dannenhoffer ${ }^{1}$ \\ ${ }^{1}$ Umpqua Community Health Center
}

July 15, 2021

\begin{abstract}
20 y/o male with history of Hodgkin's lymphoma in remission, anxiety, and depression, presented with chills, fatigue, nausea, pruritus, greasy stools, and dark urine.He had started Ashwagandha supplemntation based on recommendations by his psychiatric provider. After discontinuation, his symptoms resolved illustrating the herbs potential to cause cholestatic jaundice.
\end{abstract}

Youngest reported case of liver injury related to Ashwagandha

Simran Waller MD MPH, Roseburg Family Medicine Residency Program, swaller@umpquachc.org

Tsz Ho Wong MD, Roseburg Family Medicine Residency Program, ewong@umpquachc.org

Robert Dannenhoffer MD MPH, Roseburg Family Medicine Residency Faculty, rdannenhoffer@umpquachc.org

Funding statement: This research received no specific grant from any funding agency in the public, commercial, or not-for-profit sectors.

Conflict of interest disclosure: The authors whose names are listed have NO affiliations with or involvement in any organization or entity with any financial interest, or non-financial interest in the subject matter or materials discussed. Ethics approval statement: This research did not involve any human/animal subjects and thus did not require ethical approval from a committee or Institutional Review Board.

Patient consent statement: Verbal and written consent was obtained from the patient and legal guardian.

Material from other sources was not reproduced.

Ashwagandha, (Withania somnifera), is an herbal supplement in Ayurveda treatment. In the United States, Ashwagandha is marketed for stress reduction, brain boosting benefits, and treatment of depression. Although data on the effectiveness of this supplement is lacking, it is widely available and generally thought to be safe. However, a recent report noted 5 patients with liver injury related to Ashwagandha. Here we present a case of a young man with acute liver injury associated with its use.

A 20-year-old male with Hodgkin's lymphoma in remission, anxiety, and depression, presented to clinic with 2-weeks of generalized pruritus, dark urine, and pale, greasy bowel movements. He had associated chills, fatigue, nausea, and decreased appetite with an $8 \mathrm{lb}$ weight loss. He denied abdominal pain, scleral icterus, vomiting, or swelling. Medications included Alprazolam 0.5mg PRN, Clonazepam 1mg QHS, Desvenlafaxine ER 150mg daily, Guanfacine 1.5mg daily, Lurasidone 160mg daily, Omeprazole 20mg daily, and Risperidone $0.25 \mathrm{mg}$ PRN.

Upon further questioning, the patient disclosed that upon recommendation from his psychiatric provider, his mother ordered the "NOW" brand "standardized extract" Ashwagandha supplement 450mg via the internet as an adjuvant therapy for his anxiety. He had been taking the supplement 1-2 times daily for 4 weeks prior to the onset of symptoms. 
Physical examination showed a young male in mild distress with visible excoriations, no jaundice or organomegaly. The remainder of the exam was unremarkable. Lab work revealed normal complete blood count, ESR and electrolytes. Total bilirubin was 1.5, AST 514, ALT 955 and bile acids were elevated. A screening panel for EBV, Hepatitis A, B and C was negative.

The recent report of Ashwagandha resulting in cholestatic liver disease was noted and it was discontinued. Within two weeks, the pruritus and additional symptoms had significantly improved, and the total bilirubin and liver enzymes were down trending. One month after discontinuation, the total bilirubin was 0.4, AST 36, ALT 64 .

This is the youngest reported patient with the adverse effect of liver injury related to Ashwagandha. It emphasizes the importance of obtaining a complete history, especially herbal supplement use as their popularity increases in the United States. It also highlights the value of case series and reporting of rare side effects. Without the report in the journal that so closely matched his symptoms, the search for an alternative diagnosis may have resulted in the discontinuation of other important medications or pursuit of a more extensive workup.

Key Clinical Message:

It is important to obtain a complete history especially in terms of herbal supplement use as their popularity increases in the US. There are many rare but potential serious side effects to be aware of.

Author Contribution:

Simran Waller, MD MPH - data collection, analysis, critical revision of the article, final approval of the version Tsz Ho Wong MD - data collection, analysis, drafting the article Robert Dannenhoffer MD MPH conception, final approval of the version

References:

Bjornsson, H., Bjornsson, E., et al. May 2020. Ashwagandha as a cause for liver injury. Liver International . Volume 40, Issue 8. Pages 2035-2036. 